\title{
CLUSTER SETS ON OPEN RIEMANN SURFACES
}

\author{
MIKIO NIIMURA
}

\begin{abstract}
Generalizations of theorems of Gross-Iversen type on exceptional values are given for analytic mappings on open Riemann surfaces.
\end{abstract}

The classical, well-known theorems of Picard and Iversen (cf. [2, p. 3]) concerning isolated, essential singularities were extended to those of Hällström and Cartwright (cf. [2, pp. 10, 15]) concerning essential singularities of capacity zero, respectively. These extensions were furthermore extended to the theorems of Tsuji and Noshiro (cf. [2, pp. 14, 19]) which are stated as follows:

Let $D$ be an arbitrary domain, $B$ its boundary, $A$ a compact set of capacity zero on $B$ and $z_{0}$ a point of $A$. Let $\varphi(z)$ be single-valued and meromorphic in D. $C_{D}\left(\varphi, z_{0}\right)$ and $C_{B-A}\left(\varphi, z_{0}\right)$ denote the full clsuter set of $\varphi(z)$ at $z_{0}$ and the boundary cluster set of $\varphi(z)$ at $z_{0}$ modulo $A$, respectively.

(1) Every value of $C_{D}\left(\varphi, z_{0}\right)-C_{B-A}\left(\varphi, z_{0}\right)$ is assumed by $\varphi(z)$ infinitely of ten in any neighborhood of $z_{0}$ except for a possible set of values of capacity zero.

(2) If $\alpha \in C_{D}\left(\varphi, z_{0}\right)-C_{B-A}\left(\varphi, z_{0}\right)$ is an exceptional value of $\varphi(z)$ in a neighborhood of $z_{0}$, then either $\alpha$ is an asymptotic value of $\varphi(z)$ at $z_{0}$ or there is a sequence $\xi_{n} \in A(n=1,2,3, \ldots)$ converging to $z_{0}$ such that $\alpha$ is an asymptotic value of $\varphi(z)$ at each $\xi_{n}$.

In this paper, (1) and (2) will be generalized for analytic mappings from open Riemann surfaces into Riemann surfaces. These generalizations will be given by Theorem 1 and Theorem 2 .

Let $f$ be an analytic mapping from an open Riemann surface $R$ into a Riemann surface $S$. Let $R^{*}$ and $S^{*}$ denote a metrizable compactification and an arbitrary compactification of $R$ and $S$, respectively. $\bar{X}$ and bdy $X$ mean the closure and the boundary of a subset $X$ of $R^{*}$ or $S^{*}$ with respect to $R^{*}$ or $S^{*}$, respectively. $\partial X$ means the relative boundary of a subset $X$ of $R$ or $S$ with respect to $R$ or $S$.

We write $\Delta=R^{*}-R$. The full cluster set of $f$ at $p \in \Delta$ is defined as $C(f, p)=\bigcap_{r>0} \overline{f(U(p, r) \cap R)}$, where $U(p, r)$ denotes the $r$-neighborhood of $p$. For a set $E$ on $\Delta$, the boundary cluster set of $f$ at $p$ modulo $E$ is defined

Received by the editors February 3, 1977.

AMS (MOS) subject classifications (1970). Primary 30A72; Secondary 30A50.

Key words and phrases. Gross-Iversen's theorem, exceptional value, open Riemann surface, analytic mapping, range, asymptotic point. 
as $C_{\Delta-E}(f, p)=\bigcap_{r>0} \overline{\bigcup_{q \in W(p, r)} C(f, q)}$, where $W(p, r)=U(p, r) \cap \Delta-$ $E-\{p\}$. It is said that a path $\gamma(t)(0 \leqslant t<1)$ in $R$ tends to a connected set $K(\subset \Delta)$, when for any $r$-neighborhood $U(K, r)$ of $K$, there is a $t(K, r)$ such that $\gamma(t) \subset U(K, r)$ for all $t \geqslant t(K, r)$. Henceforth let $V(P), V_{0}(P)$ and $V^{*}(P)$ denote parametric disks about a point $P$ of $R$ or $S$.

THEOREM 1. Let $E$ be a polar set on $\Delta$ and $p$ a point of $E$. Let $r_{1}>r_{2}$ $>\cdots>r_{n}>\ldots, r_{n} \rightarrow 0$. If $E \cap$ bdy $U\left(p, r_{n}\right)=\varnothing$ for every $n$, then every point of $F=C(f, p) \cap S-C_{\Delta-E}(f, p)$ is assumed by $f$ infinitely often in any $U \in\{U(p, r)\}$, with a possible exceptional set of capacity zero.

Proof. Let $f_{U \cap R}$ denote the restriction of $f$ to $U \cap R$ and $n\left(f_{U \cap R}, b\right)$ the number of the points of $f_{U \cap R}^{-1}(b)$ for each $b \in S$, where each point is counted with its multiplicity. $n\left(f_{U \cap R}, b\right)$ is lower semicontinuous on $S$ and, hence, $F_{n}=\left\{b \in F ; n\left(f_{U \cap R}, b\right) \leqslant n\right\}(n=0,1,2, \ldots)$ is relatively closed in the open set $S-C_{\Delta-E}(f, p)$. Suppose that $\left\{b \in F ; n\left(f_{U \cap R}, b\right)<\infty\right\}$ is of positive capacity. Then there is an $N(0 \leqslant N<\infty)$ for which $F_{N-1}$ is of capacity zero and $F_{N}$ is of positive capacity, where $F_{-1}=\varnothing$. It is possible to find some $c \in F_{N}-F_{N-1}$, which is not a branch point, such that for any $V(c), V(c) \cap F_{N}$ is of positive capacity.

First consider the case where $U(p, r) \cap(\Delta-E) \neq \varnothing$ for every $U(p, r)$. Then $C_{\Delta-E}(f, p) \neq \varnothing$. Choose an open set $G(\nexists c)$ containing $C_{\Delta-E}(f, p)$. There are $V\left(a_{i}\right)(\subset U)(i=1,2, \ldots, N)$ such that $f\left(a_{i}\right)=c$ and $V\left(a_{i}\right) \cap$ $V\left(a_{j}\right)=\varnothing(i \neq j)$, and which are mapped onto a $V_{0}(c)$, satisfying $V_{0}(c) \cap G$ $=\varnothing$, by $f$ homeomorphically. There is a $U^{\prime} \in\{U(p, r)\}$ such that $U^{\prime} \subset U$ and $\overline{\cup_{q \in W} C(f, q)} \subset G$, where $W=U^{\prime} \cap \Delta-E$. For each $q \in W$, there is a $U(q) \in\{U(q, r)\}$ satisfying $f(U(q) \cap R) \subset G$. Since $f\left(\cup_{q \in W}(U(q) \cap\right.$

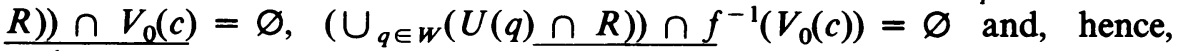
$f^{-1}\left(V_{0}(c)\right) \cap W=\varnothing$. Therefore $f^{-1}\left(V_{0}(c)\right) \cap U^{\prime} \cap \Delta \subset E$.

Choose a $U\left(p, r_{N^{*}}\right)$ such that $\overline{U\left(p, r_{N^{*}}\right)} \subset U^{\prime}$ and $\overline{U\left(p, r_{N^{*}}\right)} \cap \overline{V\left(a_{i}\right)}=\varnothing \quad(i=1,2, \ldots, N) . \overline{f\left(U\left(p, r_{N^{*}}\right) \cap R\right)}$ contains $c$, because $c \in C(f, p)$. Since $E \cap$ bdy $U\left(p, r_{N^{*}}\right)=\varnothing$, it is easy to see that $f^{-1}\left(\overline{V_{0}(c)}\right) \cap$ bdy $U\left(p, r_{N^{*}}\right)$ is compact in $R$. Hence $f\left(R \cap\right.$ bdy $\left.U\left(p, r_{N^{*}}\right)\right)$ ( $\nexists c$ ) is relatively closed on $\overline{V_{0}(c)}$. Thus it is possible to choose some $V^{*}(c)$ $\left(\subset V_{0}(c)\right)$ satisfying $\overline{V^{*}(c)} \cap f\left(R \cap\right.$ bdy $\left.U\left(p, r_{N^{*}}\right)\right)=\varnothing$.

Take a component $D^{*}\left(\subset U\left(p, r_{N^{*}}\right)\right)$ of $f^{-1}\left(V^{*}(c)\right)$. $\partial f\left(D^{*}\right)-\partial V^{*}(c)$ has regular points relative to the Dirichlet problem (cf. $[1, \mathrm{pp} .42,50])$. Let $h$ be a continuous function with the property that $h$ is equal to 0 on $\partial f\left(D^{*}\right) \cap \partial V^{*}(c)$ and $0<h \leqslant 1$ in $\partial f\left(D^{*}\right)-\partial V^{*}(c)$. Let $u$ be the solution of the Dirichlet problem in $f\left(D^{*}\right)$ with $h$ as its boundary function. Then $0<u<1$ in $f\left(D^{*}\right)$ and $\lim _{D^{*} \ni a \rightarrow q} u \circ f_{D^{*}}(a)=0$ at every $q \in \partial D^{*}$. Since $E$ is polar, there is a positive superharmonic function $s$ on $R$ with $\lim _{a \rightarrow q} s(a)=\infty$ at every $q \in E$. Therefore, for any $\varepsilon>0, \lim _{D^{*} \ni a \rightarrow q}\left(\varepsilon s_{D^{*}}(a)-u \circ f_{D^{*}}(a)\right) \geqslant 0$ at every $q \in$ $\left(\partial D^{*}\right) \cup\left(\bar{D}^{*} \cap \Delta\right)$. It follows from the minimum principle (cf. [1, p. 11]) that $-u \circ f_{D^{*}} \geqslant 0$ in $D^{*}$. This implies a contradiction. 
Next consider the case where $U(p, r) \cap(\Delta-E)=\varnothing$ for some $U(p, r)$. Then $C_{\Delta-E}=\varnothing$. We have a contradiction, as we see easily from the above proof. Thus the proof of Theorem 1 is complete.

THEOREM 2. If, under the hypotheses of Theorem $1, e \in F$ is an exceptional point of $f$ in some $U^{*} \in\{U(p, r)\}$, then either $e$ is an asymptotic point of $f$ at $p$ or there is an infinite sequence of connected sets $K_{n}(\subset E)$ converging to $p$ such that $e$ is the asymptotic point of $f$ along a path tending to $K_{n}$.

Proof. First consider the case where $U(p, r) \cap(\Delta-E) \neq \varnothing$ for every $U(p, r)$. Take any $U^{\prime \prime} \in\{U(p, r)\}$ contained in $U^{*}$. Since $n\left(f_{U^{*} \cap R}, e\right)=0$, it is possible to take $U^{\prime}, U\left(p, r_{N^{*}}\right)$ and $V^{*}(e)$ in the proof of Theorem 1 such that $\overline{U\left(p, r_{N^{*}}\right)} \subset U^{\prime} \cap U^{\prime \prime}$ and $\overline{V^{*}(e)} \cap f\left(R \cap\right.$ bdy $\left.U\left(p, r_{N^{*}}\right)\right)=\varnothing$. Any component $D^{*}\left(\subset U\left(p, r_{N^{*}}\right)\right)$ of $f^{-1}\left(V^{*}(e)\right)$ is not relatively compact in $R$.

Let $g_{V^{*}(e)}(b, e)$ denote the Green's function for $V^{*}(e)$ with pole at $e$. Suppose that $\overline{f\left(D^{*}\right)} \not \nexists e$. Then $g_{V^{*}(e)}\left(f_{D^{*}}(a), e\right)$ is bounded on $D^{*}$. As in the proof of Theorem 1 , it follows that $-g_{V^{*}(e)}\left(f_{D^{*}}(a), e\right) \geqslant 0$ in $D^{*}$. This is impossible. Therefore $\overline{f\left(D^{*}\right)} \not \nexists e$.

Let $w=\psi(b)$ be a local parameter of $V^{*}(e)$, and write $\psi\left(V^{*}(e)\right)=\{w ;|w|$ $<1\}(\psi(e)=0)$ and $W_{1 / n}=\{w ;|w|<1 / n\}(n=1,2,3, \ldots)$. Let $\left\{D_{n}\right\}$ be an infinite sequence of components of $f^{-1} \circ \psi^{-1}\left(W_{1 / n}\right)$ such that $D_{n+1} \subset D_{n}$ $\subset D^{*}$, and $\left\{p_{n}\right\}$ an infinite sequence of points $p_{n} \in D_{n}$. $D_{n}$ is not relatively compact in $R$ and $\overline{f\left(D_{n}\right)} \ni e$. For any compact set $K^{\prime}(\subset R)$, there is an $N_{0}$ such that $D_{n} \subset R-K^{\prime}$ for all $n \geqslant N_{0}$. Furthermore, as in the proof of Theorem $1, \bar{D}_{n} \subset E$. For each $n$, there is a simple arc $\lambda_{n}$ joining $p_{n}$ to $p_{n+1}$ in $D_{n}$ such that $f\left(\lambda_{n}\right) \subset \psi^{-1}\left(W_{1 / n}\right)$. Thus the path $\lambda=\cup \lambda_{n}$ tends to a component of $E \cap U\left(p, r_{N^{*}}\right)$ and has the property that $f \rightarrow e$ along $\lambda$. Since $E \cap$ bdy $U\left(p, r_{n}\right)=\varnothing$ for every $n$, our assertion is proved.

Next consider the case where $U(p, r) \cap(\Delta-E)=\varnothing$ for some $U(p, r)$. From the above proof, we see easily that our conclusion holds. Thus the proof of Theorem 2 is complete.

\section{REFERENCES}

1. C. Constantinescu and A. Cornea, Ideale Ränder Riemannscher Flächen, Springer-Verlag, Berlin and New York, 1963.

2. K. Noshiro, Cluster sets, Springer-Verlag, Berlin and New York, 1960.

Department of Mathematics, Shibaura Institute of Technology, 3-9-14, Shibaura, MinATO-KU, TOKYO, JAPAN 